\title{
Research on Application of Humanized Design in Product Design
}

\author{
Liu li-zhen \\ Wenzhou Vocational \& Technical College, Wenzhou. 325035 \\ 1918427983@qq.com
}

Keywords: Humanized; Products; Design; Application

\begin{abstract}
This paper takes soybean milk machine as an example. Nowadays, soybean milk machines on the market all adapt rotational flow to cut the beans and electric heating tube to heat. Whether they have filter nets or not or the beans are dry or wet is not the main factor to classify soybean milk machines. Instead, design is the most competitive factor. In other words, the overall design and user experience are important factors to subdivide soybean milk machines.
\end{abstract}

Figure 1 shows the family income structure of people who drink soybean milk.According to the consumer survey in 2014, different family incomes have little effect on preference of people who drink soybean milk. Structure charts of urban market levels show that in 2014 the sales volume of soybean milk machine in markets of mega cities takes $40 \%$ of the total, and mega cities are the largest markets of soybean milk machine; markets in megapolis and other markets both take half of the remaining $60 \%$ share. (Mega cities refer to cities with a population over two million; megapolis means cities with a population between 100 to 200 million; The large cities and under refer to cities with 1 million people or less).

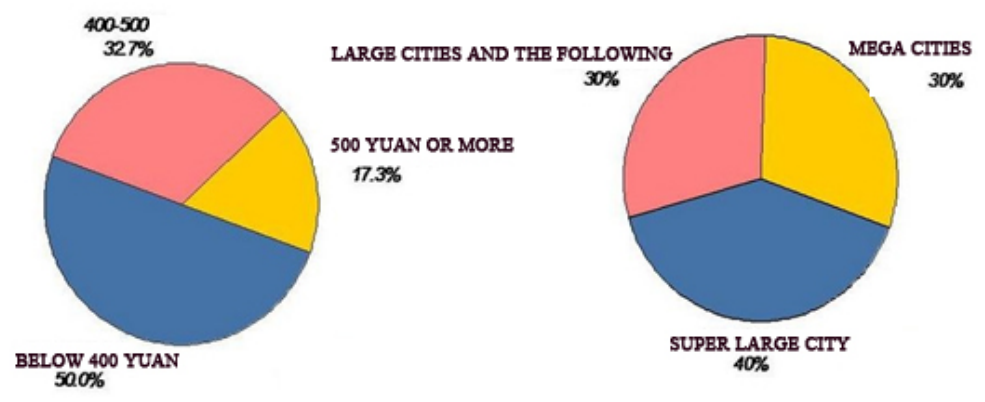

Figure 1 Yield analysis

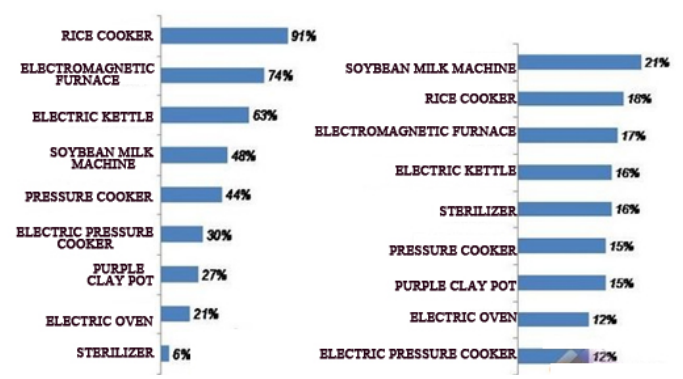

Figure 2 Classification

Figure 2 shows owning rate of small electrical appliances in urban families. Based on a consumer survey in 2014, 48\% of urban families have soybean milk machine, which is much lower than the owning rate of electric cooker and induction cooker.

In summary, it can be seen that soybean milk machine will be a necessity of our life just like TV and computer. As more and more people concern about fitness and health maintenance, there will be an increasing number of Chinese families using soybean milk machines. Less affected by age and family income, people will not pay too much attention to its price comparing to food safety and delicacy. Besides, soybean milk machine has stronger price advantage than others. Market is an important factor affecting the design, and in some time, it is the market that plays a vital role in design.

\section{Analysis on the existing soybean milk machines}

From the aspect of soybean milk machine market, Joyoung, Midea, Donlim, Philips and other brands take more than $80 \%$ of total market share. Next, soybean milk machine of these three brands-Joyoung, Philips and Midea analyzed below. 


\subsection{Analysis on structures}

As is shown in Figure 3, soybean milk machines on the market basically use this structure.In this separation structure, technical and functional components are gathered in the upper part of the soybean milk machine, and the lower part made of stainless steel is only used as a container. Control room is responsible for the entire computer-controlled process of making soybean milk. Blades are responsible for the high-speed rotation for breaking the soybeans. Level electrodes are responsible for detecting the water level to prevent dry heating. Anti-overflow electrode is responsible for detecting the water level to prevent overflow. Electrical heated tube is responsible for heating the entire soybean milk.

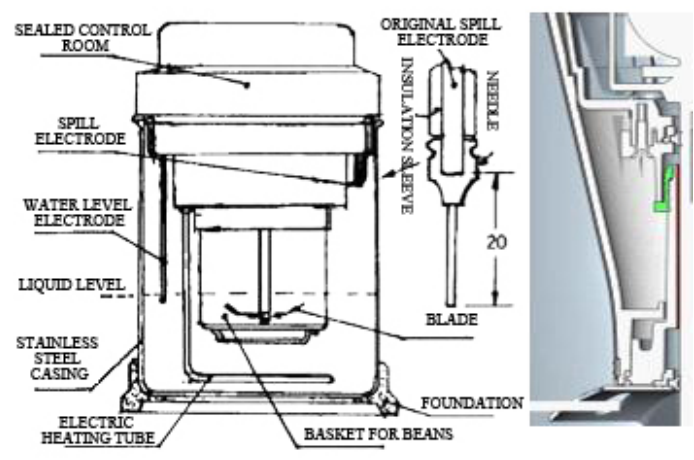

Figure 3 Analysis on structures

\subsection{Analysis on appearance}

After analysis on structure, appearance of soybean milk machines will be analyzed in the following part. Because of the separation structure, the upper part of the machine has a handle to control panel while the lower part has a side handle. Although the locations of socket holes are different, they are basically in the upper part. As a whole,the appearance of the machine honestly reflects the function and use, but the overall image of the product need to be improved because it lacks integrity and doesn't fully consider about the using process. For example, after the making of soybean milk is finished, the whole upper part has a certain temperature, but there is only one handle in the upper part, so it is clear that there is no suitable placing angle. The only solution is to put it in a bowl or on a board to wait for cleaning. All the functional opponents are gathered in the upper part, so the machine has a certain weight and is too heavy to move, so it is both inconvenient and insecure.Instead, the lower part just plays the role as a "reaction chamber"and is not made full use of.

Comparisons among soybean milk machines of brands Yoyoung, Philips and Midea indicates that although they have various styles and qualities, the basic pattern is the same, and has never been greatly improved. So there is a wide space as well as a big challenge for further efforts.

\subsection{Analysis on materials}

In aspects of the material of soybean milk machine, the material selection of the inner side is very strict because it has to withstand high temperature. The common materials of the inner side are mainly stainless steel and ABS with high-temperature resistance. As to the outside of soybean milk machine, there is a vast space for development. The appearance of the soybean milk machine is more beautiful and of finer quality. The outside materials are stainless steel, ABS and so on. Surface treatment techniques include brushed metal, electroplating, LED decoration and so forth. There is a wide-open space on exterior design.

\subsection{Analysis of Operating Mode}

The existing soybean milk machines almost adapt keys operation. Under the guidance of a given computer program, press the key to start and it needs no more time to manage. The operation mode is a direct response to functions, which need to be improved with people's higher standard for flavor. The design of operation interface is an important aspect. The operation interface is used to communicate and exchange information, so the quality of the operation interface directly determines the humanization of the product. The design could also refer to other technologies that other products 
used, such as the extensive use of LED LCD screen, and the constantly changing patterns can convey more information, are convenient for communicate, and can provide many references. Considering about the procedure of making soybean milk makes the process visualized. The human operation itself is a pleasure, just like people's joy when see the production of ice cream personally.

\subsection{Analysis on the functions}

At present, the main functions of soybean milk machine are grinding and boiling of the soybean, which are also the most direct reason for people to use and purchase this product. Besides, one of the special functions is making baby food (vegetable juice, walnut juice, and rice paste) and sesame cereal oat. To consumers, tasty soybean milk should have little bean dregs, much milk, comprehensive nutrition and strong flavor. There are a few factors in the existing soybean milk machine influencing the quality of the soybean milk, which are procedure, electrical machine, blade, heating method and grinding way. The basic law of the soybean machine existing on the market is heating the soybean milk with heating tube, and controlling the soak and boiling of the soybean and the grinding system by computer to combine them effectively in time domain and realize the making of soybean milk machine. But this leads to dependence of boiling and grinding effect on computer controlling procedures, and can not independently solve problems of the machine itself. The current soybean milk machine has already had many functions, realized “one machine, multiple usages", and gained excellent market implication. But it lacks professional spirit in product concept.

\subsection{Analysis on the environment}

When the soybean milk machine is working, there is a lot of noise. It is not as gentle as it seems to be, and the sound is very loud. This is due to the operating principle of the soybean milk machine, and interaction between the soybean and the blade will definitely generates noises. But this factor should be considered into the design process of the products. Given the using environment of household appliances-the space for people to live, factors which may affect people's life should be considered. The design of the products should be responsible for consumers.
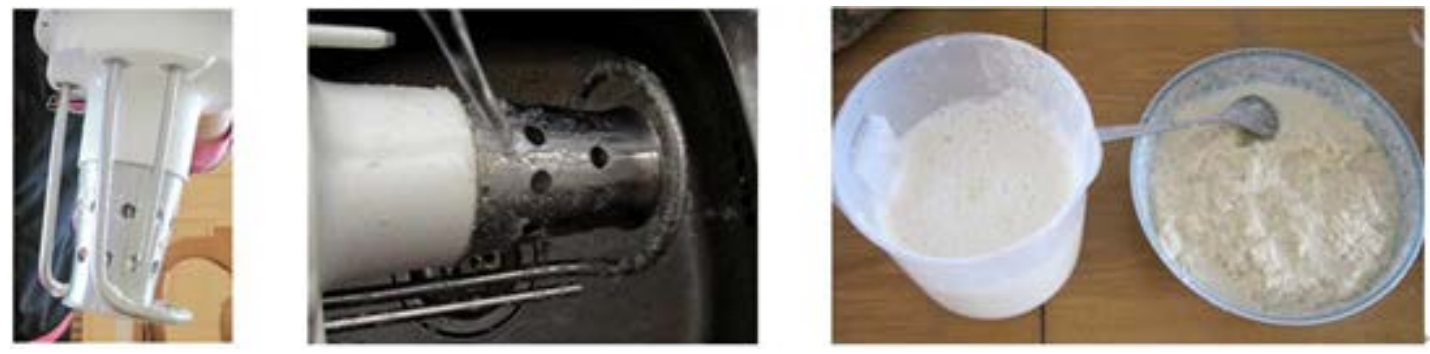

Figure 4 Machine head

Now, let's talk about the placement problem of the machine head. When the making of soybean milk is finished, the heating tube and the plastic part of ABS have a certain temperature, and should never be placed casually. But almost all the current soybean milk machines don't consider about this problem. Figure 4, clean problem. The machine head part (the upper part) has a certain height. When cleaning, if we carry it by one hand, and use the other hand to clean, we will feel very painful. Because soybean has a certain level of adhesiveness, so it is not easy to be cleaned as the ads say "clean by one wash". So the part needing washing should be improved.

\section{Conclusion of the market survey}

Soybean is a kind of drink with Chinese features. Its making should trace its source, adapting the most proper method. The pure flavor and complete nutrients of the soybean milk should be ensured. To meet peoples' demands in the fast-paced life, the current soybean milk machine has become very excellent. Now we don't need to do repetitive work, but should find out a new path and new land to expand, and building the product image of soybean milk machine. 


\section{Project design}

This project, starting with the cultural feelings, taking petals as the source of inspiration, changes and summarizes things about this machine. It considers about the sense of elegant texture when grinding, so the shapes are exaggerated and the practicability is not very high, and there emerges many problems when refining.

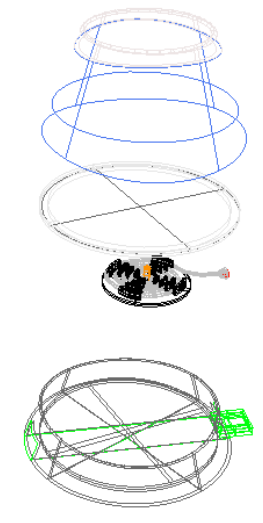

Figure 5 Structure

Figure 5 Diversified sources of inspiration. This design makes people feel powerful as the ancient bottles do. Spirals of smoke bring people the feeling of elegant and it contains the form of Oracle. By overall consideration, this design is settled to be the final project.

\section{Project description}

\subsection{Product structure}

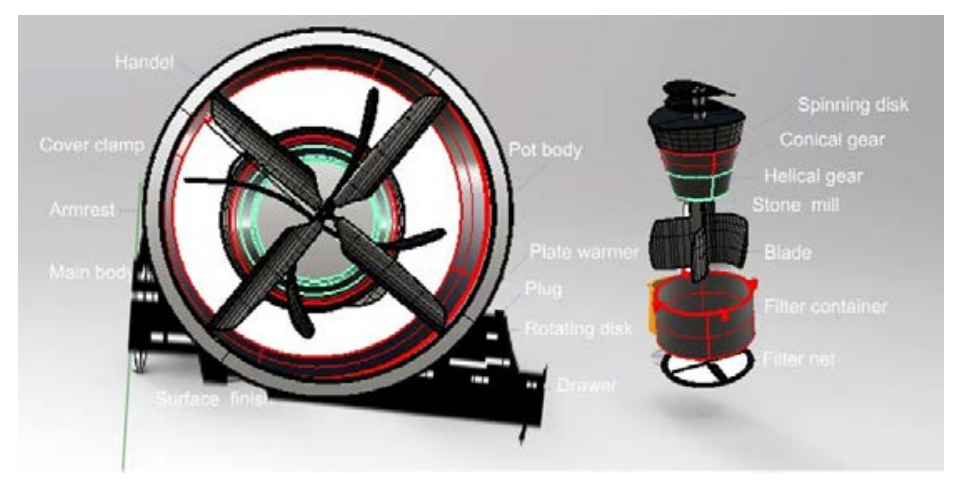

Figure 6 Structure chart

The appearance in Figure 6 is derived from the functions, obeying the "form follows function" rule. Because the "form" is for grinding, anything should be considered around the noun "grinding" and the verb "grind". It is convenient, comfortable, pleasing and elegant for the operation. The main body uses the shape and structure of a wine goblet, because the wine goblet is generous and stable. In balanced shape, it adapts the curve sense of long sleeves and curling smoke, realizing the combination of softness and hardness. This design also makes some changes in aspect of texture. It tries to realize both the classical and modern effect in the meantime.

\subsection{Analysis on inner structure of products}

The soybean milk machine has refined appearance as well as well-designed inner structure. In functions, it can be divided into two parts which are soybean grinding and bean dregs filtering. The soybean grinding process adapts layer-upon-layer progressive form. First, the soybean is pushed downwards by a spinning plate; conical insert grinds the soybean preliminarily; helical teeth push the soybean dregs downwards; stone grinding head accurately grinds the soybean. The upside of the flabellum coordinates with the stone grinding head, and the bottom coordinates with support pillar of the filter tank. Because the soybean dregs may block the filter mouth, the flabellum-sweeping method 
is adapted to ensure that the soybean dregs can be filtered smoothly. Analysis on product size is shown in Figure 7.

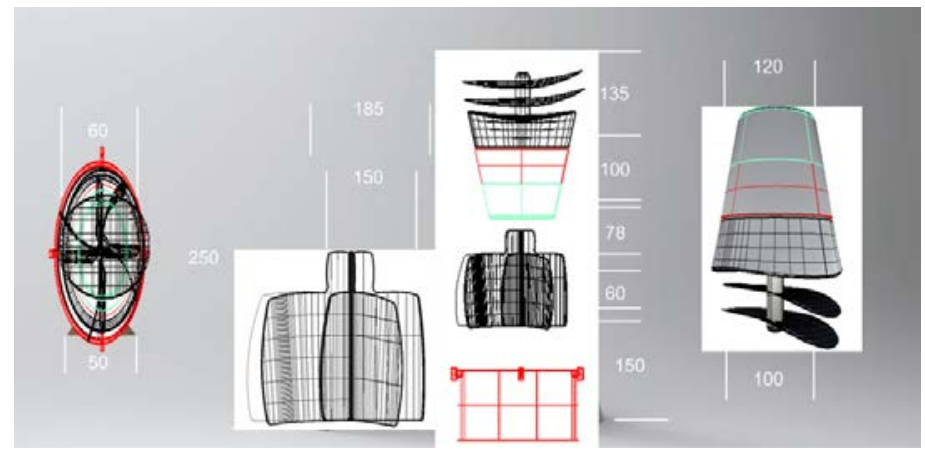

Figure 7 Analysis on product size

Leaving the cable outside affects the beauty and operation of soybean milk machine, so the cable management device is designed. because it's easy for bean dregs to block the filter screen, filter unit is designed.

\section{Conclusion}

Application of design concepts in design practice needs people's constant exploration. The soybean milk machine, as the symbol of soybean milk culture, should embody the wisdom of oriental design in aspects of appearance and using process, and it contains Chinese cultural philosophy. With the development of electrification and manufacture technology, the electric appliances change their "white box operation" into "black box operation", which means all of the machine structures and functional components are all hidden under the appearance. People know little about machines' working rules but they have to learn them. To this kind of contradiction, more products turn to the display screen for help, but this is not the only way. So this design explores the solution to this problem from the perspective of product semantics, making the products speak by themselves, namely having a certain level of self-evidence. Semantics of products is absorbed from Chinese traditional culture, endowing the soybean milk machine some peculiarity of Chinese design. And this design also makes best efforts to integrate many other ideas, so there will not be any pities.

\section{References}

[1] ZHAO Jiang-hong.The Im plication of Design Art [M]. Chang-sha Hunan University Publishing House, 2005. [3]

[2] CROSS N. Designer Ways Knowing[M].SHEN Hao-xiang,REN Wen-yong,CHEN Shi,Translate. Wuhan: Huazhong Uni-versity of Science\& Technology Press,2013.

[3] Zhiliang Xia. Application of reverse engineering based on computer in product [J] . International Journal of Multimedia and Ubiquitous Engineering, 2014,9(5); 343-354

[4] Zhiliang Xia. Design Research on Electronic Learning Products Based on “People-oriented” [J] . International Journal of Multimedia and Ubiquitous Engineering, 2014,9(4);209-220 